\title{
Association between insulin-like growth factor 1 and insulin resistance in obese prepubertal boys:a cross-sectional study
}

\section{Jiangying Kuang}

the second hospital,Cheeloo College of Medicine

\section{Li Zhang}

the first Affiliated hospital of shandong First Medical University

\section{Yueqin Xu}

The First Affiliated Hospital of Chongqing Medical University

\section{Jiang Xue}

The Second Honspital, Cheeloo College Of Medicine Shandong University

\section{Shuang Liang ( $\square$ liangshuang3508@163.com )}

Second Hospital of Shandong University https://orcid.org/0000-0001-9067-6829

\section{Research article}

Keywords: Insulin like growth factor 1, Insulin resistance, Whole body insulin sensitivity index, Obesity, Prepubertal boys

Posted Date: December 1st, 2020

DOl: https://doi.org/10.21203/rs.3.rs-114624/v1

License: (c) (i) This work is licensed under a Creative Commons Attribution 4.0 International License. Read Full License 


\section{Abstract}

Background As one of the most common features of obesity, insulin resistance is central to the pathogenesis of the metabolic syndrome. Low insulin-like growth factor 1(IGF-1) levels have been proven to be associated with many traditional cardiovascular risk factors, but it still remains controversy with the relationship between IGF-1 and insulin resistance. Accordingly, the main purpose of this study is to investigate the relationship between IGF-1 and insulin resistance in obese prepubertal boys.

Methods We used whole body insulin sensitivity index (WBISI) to represent insulin resistance. 70 obese prepubertal boys were included in this study, and the obese subjects were divided into two groups by using 1.285 as a threshold value for WBISI. Clinical examination and laboratory examinations were assessed for all participants.

Results Among obese boys, the group of children with WBISI $\leq 1.285$ had lower IGF- 1 standard deviation scores (SDS) $(p=0.021)$ than WBISI $>1.285$ group. The results of multivariate stepwise regression analysis show that WBISI was positively correlated with IGF-1 SDS $(\beta=1.726, p=0.002)$ after adjusting for traditional cardiovascular risk factors.

Conclusion IGF-1 SDS was negatively associated with insulin resistance in obese prepubertal boys, independent of other traditional cardiovascular disease risk markers.

\section{Background}

Childhood obesity has become major health problems all over the world. It is not only an independent chronic metabolic disease, but also an important risk factor for chronic diseases such as hypertension, hyperlipidemia, type 2 diabetes and metabolic syndrome in children[1]. As the most common feature of childhood obesity, insulin resistance is critical to the pathogenesis of the metabolic syndrome. Several quantitative tools have been used to assess insulin sensitivity and insulin resistance, but there is still not consensus of standards in the pediatric population. Although hyperinsulinemic-euglycemic clamp and the frequently sampled intravenous glucose tolerance test were described as gold standard methods for assessing insulin resistance, it is impractical to be widely used in pediatric population due to its expensive and multiple blood drawn. Homeostatic model assessment index of insulin resistance (HOMAIR), a simpler, less invasive method, has been widely used in children and adolescents, however, Shaibi et al[2] showed that fasting indices is not recommended when studying the effect of interventions on insulin sensitivity in overweight youth. The whole body insulin sensitivity index (WBISI) derived from an oral glucose tolerance test (OGTT) provides reasonable estimates of insulin sensitivity and insulin resistance, and has been validated as good surrogate measures of insulin resistance in obese children and adolescents[3, 4].

Insulin-like growth factor-1 (IGF-1) is primarily produced in the liver upon stimulation by growth hormone $(\mathrm{GH})$, and it plays key roles in regulating proliferation, differentiation, metabolism and cell survival[5]. There were lots of accumulating evidences that obese children and experimental animals were often 
accompanied with low IGF-1 levels[6, 7]. Moreover, low IGF-1 levels were independently associated with cardiovascular risk factors such as nonalcoholic fatty liver disease[8], low high-density lipoprotein cholesterol[6] and metabolic syndrome[6] in obese children. Nonetheless, it still remains controversy with the relationship between IGF-1 and insulin resistance. Some research showed low circulating IGF-1 level was independently associated with hyperglycemia and insulin resistance in adults[9-11]. However, Ushaped curve[12] or no significant correlation[6] was noted between IGF-1 levels and HOMA-IR in other studies. Furthermore, most previous studies used adults as main samples, and also most of them just used HOMA-IR to represent insulin resistance. To best of our knowledge, no study have ever used WBISI to represent insulin resistance to assess the relationship between IGF-1 and insulin resistance in children. To this end, this study aimed to investigate the association between IGF-1 and WBISI in obese prepubertal boys.

\section{Materials And Methods}

\section{Subjects}

We recruited 70 obese prepubertal boys (age 7-12 year) who had been referred to the Department of Pediatrics, The Second Hospital, Cheeloo College of Medicine, Shandong University. All subjects were obese (Body mass index (BMI) $₫ 95$ th percentile for the age and sex), but otherwise healthy. Exclusion criteria included (1) any syndrome or disease that could influence dietary intake and endocrine disorders; (2) type 1 or 2 diabetes mellitus; (3) serious infection, systemic disease and other chronic wasting illnesses; (4) short stature or the growth velocity is less than $5 \mathrm{~cm} /$ year; (5) the use of medication that would influence body composition, GH secretion or blood pressure, glucose or lipid metabolism.

Taking WBISI as the main variable, obese children were classified according to the median WBISI values to find the center value for this population of obese children. Thus, the obese children were divided into two groups: obese children with a WBISI $\leq$ median value and obese children with a WBISI > median value.

The Ethics Committee of The Second Hospital, Cheeloo College of Medicine, Shandong University approved the study. Written informed consent was obtained from all parents and subjects.

\section{Anthropometric Measurements}

Body weight was determined to the nearest $0.1 \mathrm{~kg}$ on a standard electronic scale and height was measured with standard height stadiometer to the nearest $0.1 \mathrm{~cm}$. BMI was determined as weight $/$ height $^{2}$ and expressed as $\mathrm{kg} / \mathrm{m}^{2}$. Body mass index standard deviation score (BMI-SDS) were calculated based on the age and sex reference values for Chinese children[13]. Pubertal stage according to Tanner criteria[14]. Blood pressure was measured with Audio Intelligent Electronic Sphygmomanometer (HEM-7071, OMRON, China) after a 30-min rest, in a supine position. Two measurements were made, and record the average of two measurements of systolic blood pressure (SBP) and diastolic blood pressure (DBP).

\section{Laboratory Measurements}


Fasting blood samples were collected from subjects after a 12-h overnight fast for measurement of endocrine indexes, glucose, lipids levels and other metabolic factors. OGTT was performed. Plasma glucose and insulin values were assessed at time 0, and 30, 60, 90 and 120 minutes after the consumption of an oral glucose solution $(1.75 \mathrm{~g} / \mathrm{kg}$, maximum of $75 \mathrm{~g})$. Free triiodothyronine (FT3), free thyroxine(FT4), thyroid-stimulating hormone (TSH), adrenal corticotropic hormone (ACTH), cortisol (COR) and IGF-1 were measured using chemiluminescence assay (Siemens Healthcare Diagnostics, USA). IGF-1 levels were transformed into IGF-1 SDS based on the age-gender related normative references[15].Total cholesterol(TC), high density lipoprotein cholesterol (HDL-C), low density lipoprotein-cholesterol(LDL-C), triglycerides(TG), fasting plasma glucose (FPG) and uric acid were detected by using an Auto Biochemical Analyzer (AU5400, Beckman Coulter, Tokyo, Japan). Fasting insulin was measured with chemiluminescent immunometric assays (CobasE170, Roche Diagnostics, Mannheim, Germany). Plasma glycosylated haemoglobin (HbA1c) was measured using high performance liquid chromatography (Tosoh Corporation, Tokyo, Japan).Insulin resistance was estimated using WBISI. WBISI $=10,000$ /square root of [fasting glucose $\times$ fasting insulin $\{\mu \mathrm{U} / \mathrm{mL}\}] \times[$ mean glucose $\{\mathrm{mg} / \mathrm{dL}\} \times$ mean insulin during $\operatorname{OGTT}\{\mu \mathrm{U} / \mathrm{mL}\}][16]$.

\section{Statistical analysis}

We used the Statistical Package for Social Sciences, version 20.0 (SPSS Inc. Chicago, USA) to analyze our data. The data were expressed as the mean $( \pm S D)$ of normally distributed or median (interquartile range) of skewed data. Data that were not normally distributed were transformed logarithmically for analysis. Differences in continuous variables between two groups were assessed by Student's t-test for normally distributed data, variables which cannot be transformed to normal distribution were analyzed by the Mann-Whitney $U$ test. A stepwise multiple linear regression analysis was performed to test the associations of IGF-1 SDS (the dependent variable) with independent variables including WBISI, BMI SDS and the other metabolic risk factors (blood pressure, TG, HDL-C, LDL-C, FBG and uric acid) to determine whether the association between IGF-1 SDS and insulin resistance was independent of obesity or other metabolic markers. A p value of $<0.05$ was considered statistically significant.

\section{Results}

The median WBISI value for the population studied was 1.285. The data of the children with WBISI $\leq$ 1.285 ( 35 children) were compared with the data of the children with WBISI >1.285 ( 35 children).

The characteristics of the anthropometric and clinical features were shown in Table 1. No significant differences were found in age. BMI SDS was similar in the two groups. Blood pressure values were the same in both groups. 
Table 1

Clinical characteristics in the two groups according to WBISI levels

\begin{tabular}{|llll|}
\hline Variable & WBISI $\leq 1.285(n=35)$ & WBISI $>1.285(n=35)$ & $p$ value \\
\hline Age $(y r)$ & $9.95 \pm 110$ & $9.99 \pm 1.30$ & 0.902 \\
\hline BMI SDS & $3.35 \pm 0.91$ & $3.12 \pm 0.16$ & 0.298 \\
\hline SBP $(\mathrm{mmHg})$ & $125.57 \pm 15.49$ & $125.40 \pm 13.57$ & 0.961 \\
\hline DBP $(\mathrm{mmHg})$ & $81.54 \pm 11.74$ & $78.14 \pm 12.03$ & 0.236 \\
\hline * p $\otimes 0.05$ & & \\
\hline $\begin{array}{l}\text { Abbreviations: BMI SDS, Body mass index standard deviation scores; SBP, Systolic blood pressure; } \\
\text { DBP, Diastolic blood pressure. }\end{array}$ & \\
\hline
\end{tabular}

In obese children, pituitary-thyroid axis, pituitary-adrenal axis, and IGF-1 have been performed. Endocrine factors findings of the obese children were stratified according to WBISI, which were shown in Table 2. Notably, the group of children with WBISI $\leq 1.285$ had lower IGF-1 SDS $(p=0.021)$. No significant differences were seen in the levels of FT3, FT4, TSH, ACTH and COR in the two groups.

Table 2

Endocrine factors in the two groups according to WBISI levels

\begin{tabular}{|c|c|c|c|}
\hline Variable & WBISI $\leq 1.285(n=35)$ & WBISI >1.285 $(n=35)$ & $p$ value \\
\hline IGF-1SDS & $-1.08 \pm 1.78$ & $-0.22 \pm 1.25$ & $0.021^{*}$ \\
\hline FT3 (pmmol/L) & $6.37 \pm 0.96$ & $6.07 \pm 0.72$ & 0.151 \\
\hline FT4 (pmmol/L) & $16.20(14.67-17.34)$ & $14.69(12.24-16.72)$ & 0.068 \\
\hline $\mathrm{TSH}(\mu \mathrm{U} / \mathrm{mL})$ & $2.94(1.87-3.42)$ & $3.79(2.02-5.40)$ & $0.076 \#$ \\
\hline ACTH $(\mathrm{mmol} / \mathrm{L})$ & $31.85(17.47-49.24)$ & $22.80(16.46-39.29)$ & $0.144 \#$ \\
\hline $\mathrm{COR}(\mathrm{pg} / \mathrm{mL})$ & $330.00(264.30-534.00)$ & $360.00(241.00-453.00)$ & 0.539 \\
\hline \multicolumn{4}{|c|}{ \# Mann-Whitney U test * $\mathrm{p} \llbracket 0.05$} \\
\hline \multicolumn{4}{|c|}{$\begin{array}{l}\text { Abbreviations: IGF-1 SDS, Insulin-like growth factor } 1 \text { standard deviation scores; FT3, Free } \\
\text { triiodothyronine; FT4, Free thyroxine, TSH, Thyroid-stimulating hormone; ACTH, Adrenal corticotropic } \\
\text { hormone; COR, Cortisol }\end{array}$} \\
\hline
\end{tabular}

Table 3 shows that the levels of cardiovascular metabolic risk factors of the obese children stratified according to WBISI. No significant differences were seen in the levels of TC, HDL-C, LDL-C, TG and uric acid between the two groups. In addition, there were no significant differences in FBG and HbA1C. 
Whereas higher insulin levels $(p<0.001)$ were observed in the subjects with WBISI $\leq 1.285$ than in children with WBISI >1.285.

Table 3

Cardiovascular metabolic risk factors according to WBISI levels in obese children

\begin{tabular}{|c|c|c|c|}
\hline Variable & $W B|S| \leq 1.285(n=35)$ & WBISI >1.285 $(n=35)$ & $p$ value \\
\hline $\mathrm{TC}(\mathrm{mmol} / \mathrm{L})$ & $4.31(3.77-4.68)$ & $4.16(3.56-4.67)$ & 0.156 \\
\hline $\mathrm{HDL}-\mathrm{C}(\mathrm{mmol} / \mathrm{L})$ & $1.11 \pm 0.21$ & $1.12 \pm 0.24$ & 0.850 \\
\hline LDL-C (mmol/L) & $2.64(2.26-2.99)$ & $2.51(16.46-39.29)$ & 0.162 \\
\hline $\mathrm{TG}(\mathrm{mmol} / \mathrm{L})$ & $1.39(0.89-2.22)$ & $1.30(0.85-1.75)$ & 0.557 \\
\hline Uric acid $(\mu \mathrm{mol} / \mathrm{L})$ & $422.49 \pm 102.33$ & $404.78 \pm 131.30$ & 0.531 \\
\hline Insulin $(\mu \mathrm{IU} / \mathrm{mL})$ & $52.17(39.20-60.70)$ & $17.40(14.10-26.36)$ & $\mathrm{p} \otimes 0.001^{\star}$ \\
\hline FBG (mmol/L) & $5.24 \pm 0.67$ & $4.99 \pm 0.47$ & 0.078 \\
\hline HbA1c (\%) & $5.90(5.70-6.30)$ & $5.80(5.50-6.00)$ & $0.473 \#$ \\
\hline \multicolumn{4}{|c|}{ \# Mann-Whitney U test * p $\mathbb{0} 0.05$} \\
\hline
\end{tabular}

According to the results of the multivariate stepwise regression analysis, Ig WBISI was positively correlated with IGF-1 SDS $(\beta=1.726, p=0.002)$. And the results of multiple regression analysis showed that Ig WBISI was the only variable that explained the IGF-1 SDS after adjusting for BMI SDS, SBP, DBP, HDL-C, TG, LDL-C, FBG and uric acid.

\section{Discussion}

Our study found that reduced IGF-1 were present in obese prepubertal boys with lower values of WBISI, which is a surrogate marker of insulin resistance. Furthermore, we provided evidences that IGF-1 SDS was significantly correlated with WBISI after controlling for BMI SDS, blood pressure and other metabolic risk factors.

In the study, we confirmed a significant association between low levels of IGF-1 and insulin resistance in obese prepubertal boys. Moreover, the results of multiple regression analysis showed that insulin resistance was the only variable that explained the IGF-1 SDS after adjusting for other traditional cardiovascular disease risk markers. Based on the results, we can infer that the correlation between IGF-1 and traditional cardiovascular disease may be mediated by insulin resistance, thus it represents a possible direction for a forthcoming research and therapeutic approach. 
There is still not very clear about the precise underlying mechanisms of the correlation between IGF-1 and insulin resistance. The possible reasons of this correlation may be related to the following factors. Insulin and IGF-1 share structural homology, interact with the same membrane receptors with different affinities to mediate a wide range of metabolic and growth-promoting functions. Studies have shown that insulin was likely to decrease IGF-1 through differential modulation of IGF binding proteins[17]. This may be one mechanism for the link of IGF-1 and insulin resistance. Another possible explanations for the association between IGF-1 and insulin resistance are driven by chronic inflammatory. Low levels of IGF-1 in the C3H.6T mice [18] and in the liver-specific IGF-1 knockout mouse [19]were associated with enhanced inflammatory phenotype. On the contrary, elevated IGF-1 levels could attenuate the anti-inflammatory effects and the lowered oxidative stress[19-21]. Meanwhile, inflammatory factors and chronic inflammatory responses play an important role in the occurrence and development of insulin resistance[22].

In addition, macrophages may also be involved in the complex process of interaction between IGF-1 and insulin. Insulin has been considered be pro-inflammatory in macrophages[23] and has been reported to promote foam cell formation[24]. By contrast, recent research showed that IGF-1 might have a fundamental role in macrophage activation $[25,26]$ and had the anti-inflammatory ${ }^{26}$ and antifoam cell formation[27] effects. Further studies are necessary to clarify specific mechanisms and effects of IGF-1 and insulin on macrophages.

This study also has several potential limitations. Firstly, we have not applied hyperinsulinemiceuglycemic clamp which is considered to be the gold standard for assessing insulin resistance. However, the clamp technique is invasive, expensive and not suitable for child clinical practice. Secondly, it is a cross-sectional study, so the causal relationship between IGF-1 and insulin resistance cannot be clarified. Lastly, our sample was limited to Chinese obese prepubertal boys, but gender and pubertal developmental stage restriction can also reduce confounding factors. In spite of these limitations, to best of our knowledge, this study is one of the first attempt to report the association between IGF-1 and WBISI in obese children, thus it should have some implications for clinical practice. Our data suggested that the assessment of IGF-1, perform OGTT experiments and calculate WBISI might provide useful clinical information on the severity and complications of obesity in children.

In conclusion, we have found a strong correlated relationship between low IGF-1 and insulin resistance in obese prepubertal boys independently of other risk factors. The role of IGF-1 needs further examination whether it is a potential therapeutic target in obese patients of increased cardiometabolic risk or only an indicator of metabolic disorders. And further studies are needed to determine the characterize of the pathophysiology of the association between IGF-1 and insulin resistance in obese children and obesityrelated complications.

\section{Abbreviations}


HOMA-IR, Homeostasis model assessment-insulin resistance; OGTT, Oral glucose tolerance test; WBISI, Whole body insulin sensitivity index; IGF-1, Insulin-like growth factor 1; GH, Growth hormone; BMI, Body mass index; BMI SDS, Body mass index standard deviation scores; SBP, Systolic blood pressure; DBP, Diastolic blood pressure; FT3, Free Friiodothyronine; FT4, Free thyroxine, TSH, Thyroid-stimulating hormone; ACTH, Adrenal corticotropic hormone; COR, Cortisol; TC, Total cholesterol; HDL-C, High density lipoprotein cholesterol; LDL-C, Low density lipoprotein cholesterol; TG, Triglycerides; FBG, Fasting blood glucose; HbA1C, Glycosylated hemoglobin;

\section{Declarations}

Acknowledgements:The authors are grateful to all children and their parents for participating in this study.

Funding:This research was supported by Jinan clinical medical science and technology innovation plan (NO. 201907002), National Natural Science Foundation of China (NO. 81800775), Doctoral Fund of Shandong Provincial Natural Science Foundation, China (ZR2018BH010) and Youth Fund of the Second Hospital of Shandong University (2018YT21).

Authors' Contributions: SL designed the study, performed the data analysis, and drafted the initial manuscript. JK critically reviewed and revised the manuscript. LZ and YX contributed significantly to revision of the manuscript. JX performed the data collection. All authors read and approved the final manuscript.

Availability of data and materials:The datasets used and/or analysed during the current study are available from the corresponding author on reasonable request.

Conflict of Interest!The authors have no conflicts of interest to declare.

Ethics approval and consent to participate:The study protocol was approved by the Ethics Committee of The Second Hospital, Cheeloo College of Medicine, Shandong University (No.KYLL-2017(LW)001). All the patients and their parents gave their written information consent.

\section{References}

1. Skinner AC, Perrin EM, Moss LA, Skelton JA. Cardiometabolic Risks and Severity of Obesity in Children and Young Adults. N Engl J Med 2015; 373: 1307-1317

2. Shaibi GQ, Davis JN, Weigensberg MJ, Goran MI. Improving insulin resistance in obese youth: choose your measures wisely. Int J Pediatr Obes 2011; 6: e290-296

3. Brar PC, Koren D, Gallagher PR, Pendurthi B, Katz LE. Comparison of oral and intravenous glucose tolerance test derived sensitivity and secretory indices in obese adolescents. Clin Pediatr (Phila) 2013; 52: 247-253 
4. Yeckel CW, Weiss R, Dziura J, Taksali SE, Dufour S, Burgert TS, Tamborlane WV, Caprio S. Validation of insulin sensitivity indices from oral glucose tolerance test parameters in obese children and adolescents. J Clin Endocrinol Metab 2004; 89: 1096-1101

5. Troncoso R, Ibarra C, Vicencio JM, Jaimovich E, Lavandero S. New insights into IGF-1 signaling in the heart. Trends Endocrinol Metab 2014; 25: 128-137

6. Liang S, Hu Y, Liu C, Qi J, Li G. Low insulin-like growth factor 1 is associated with low high-density lipoprotein cholesterol and metabolic syndrome in Chinese nondiabetic obese children and adolescents: a cross-sectional study. Lipids Health Dis 2016; 15: 112

7. Ren J, Anversa P. The insulin-like growth factor I system: physiological and pathophysiological implication in cardiovascular diseases associated with metabolic syndrome. Biochem Pharmacol 2015; 93: 409-417

8. Liang S, Cheng X, Hu Y, Song R, Li G. Insulin-like growth factor 1 and metabolic parameters are associated with nonalcoholic fatty liver disease in obese children and adolescents. Acta Paediatr 2017; 106: 298-303

9. Mannino GC, Greco A, Lorenzo CD, Andreozzi F, Marini MA, Perticone F, Sesti G. A Fasting InsulinRaising Allele at IGF1 Locus is Associated with Circulating Levels of IGF-1 and Insulin Sensitivity. PLoS One 2013; 8: e85483

10. Teppala S, Shankar A. Association Between Serum IGF-1 and Diabetes Among U.S. Adults. Diabetes Care 2017; 33: 2257-2259

11. Lam CS, Chen MH, Lacey SM, Yang Q, Sullivan LM, Xanthakis V, Safa R, Smith HM, Peng X, Sawyer DB, Vasan RS. Circulating insulin-like growth factor-1 and its binding protein-3: metabolic and genetic correlates in the community. Arterioscler Thromb Vasc Biol 2010; 30: 1479-1484

12. Friedrich $N$, Thuesen $B$, Jorgensen $T$, Juul A, Spielhagen $C$, Wallaschofksi $H$, Linneberg $A$. The Association Between IGF-I and Insulin Resistance: A general population study in Danish adults. Diabetes Care 2012; 35: 768-773

13. Li H, Ji CY, Zong XN, Zhang YQ. Height and weight standardized growth charts for Chinese children and adolescents aged 0 to 18 years. Chin J Pediatrics 2009; 47: 487-92.

14. Tanner JM, Whitehouse RH. Clinical longitudinal standards for height, weight, height velocity, weight velocity, and stages of puberty. Arch Dis Child 1976; 51: 170-179

15. Isojima T, Shimatsu A, Yokoya S, Chihara K, Tanaka T, Hizuka N, Teramoto A, Tatsumi K-i, Tachibana K, Katsumata N. Standardized centile curves and reference intervals of serum insulin-like growth factor-I (IGF-I) levels in a normal Japanese population using the LMS method. Endocrine journal 2012; 59: 771-780

16. Matsuda M, Defronzo RA. Insulin sensitivity indices obtained from oral glucose tolerance testing: comparison with the euglycemic insulin clamp. Diabetes Care 1999; 22: 1462

17. Akanji AO, Smith RJ. The insulin-like growth factor system, metabolic syndrome, and cardiovascular disease risk. Metab Syndr Relat Disord 2012; 10: 3-13 
18. Shai SY, Sukhanov S, Higashi Y, Vaughn C, Rosen CJ, Delafontaine P. Low circulating insulin-like growth factor I increases atherosclerosis in ApoE-deficient mice. Am J Physiol Heart Circ Physiol 2011; 300: H1898-1906

19. Sivasubramaniyam T, Schroer SA, Li A, Luk CT, Shi SY, Besla R, Dodington DW, Metherel AH, Kitson AP, Brunt JJ, Lopes J, Wagner KU, Bazinet RP, Bendeck MP, Robbins CS, Woo M. Hepatic JAK2 protects against atherosclerosis through circulating IGF-1. JCI Insight 2017; 2

20. Sukhanov S, Higashi Y, Shai SY, Blackstock C, Galvez S, Vaughn C, Titterington J, Delafontaine P. Differential requirement for nitric oxide in IGF-1-induced anti-apoptotic, anti-oxidant and antiatherosclerotic effects. FEBS Lett 2011; 585: 3065-3072

21. Jeschke MG, Barrow RE, Herndon DN. Insulinlike Growth Factor I Plus Insulinlike Growth Factor Binding Protein 3 Attenuates the Proinflammatory Acute Phase Response in Severely Burned Children. Ann Surg 2000; 231: 246-252

22. Shoelson SE, Herrero L, Naaz A. Obesity, Inflammation, and Insulin Resistance. Gastroenterology 2007; 132: 2169-2180

23. Manowsky J, Camargo RG, Kipp AP, Henkel J, Püschel GP. Insulin-induced cytokine production in macrophages causes insulin resistance in hepatocytes. Am J Physiol Endocrinol Metab 2016; 310: E938-E946

24. Park YM, R Kashyap S, A Major J, Silverstein RL. Insulin promotes macrophage foam cell formation: potential implications in diabetes-related atherosclerosis. Lab Invest 2012; 92: 1171-1180

25. Higashi Y, Sukhanov S, Shai S-Y, Danchuk S, Tang R, Snarski P, Li Z, Lobelle-Rich P, Wang M, Wang D. Insulin-Like Growth Factor-1 Receptor Deficiency in Macrophages Accelerates Atherosclerosis and Induces an Unstable Plaque Phenotype in Apolipoprotein E-Deficient Mice. Circulation 2016; 133 : 2263-78

26. Yusuke, Higashi, Sandeep, Gautam, Patrick, Delafontaine, Sergiy, Sukhanov. IGF-1 and cardiovascular disease. Growth Horm IGF Res 2019; 45: 6-16

27. Sukhanov S, Snarski P, Vaughn C, Lobelle-Rich P, Kim C, Higashi Y, Shai S-Y, Delafontaine P. Insulinlike growth factor I reduces lipid oxidation and foam cell formation via downregulation of $12 / 15$ lipoxygenase. Atherosclerosis 2015; 238: 313-320 